\title{
Children Spend Less Time Playing with Gender-Typical Toys These Days
}

\author{
John A. Barry ${ }^{1}$ (1) $\cdot$ Brenda K. Todd ${ }^{2}$ \\ Received: 11 June 2020 / Revised: 30 December 2020 / Accepted: 13 January 2021 / Published online: 4 February 2021 \\ (c) The Author(s), under exclusive licence to Springer Science+Business Media, LLC part of Springer Nature 2021
}

The February 2020 issue of Archives of Sexual Behavior published a very interesting study of sex differences in toy preference (Davis \& Hines, 2020). The paper followed the findings from an earlier meta-analysis/meta-regression by ourselves (Todd et al., 2018), and Davis and Hines are to be congratulated on assessing a wider range of protocols for assessing toy preference (free play, visual preference, forced choice, and naturalistic approaches) than we used in our paper, which assessed free play only.

We would like to highlight a claim made by Davis and Hines regarding the impact of historical time (measured by study publication year) on play behavior. Unlike Todd et al. (2018), Davis and Hines (2020) say they found no such impact. However, Davis and Hines did not appear to assess correlations between the amount of time (measured in seconds) children spent in play with male-typed toys and femaletyped toys, and the duration of time since publication of the study. We found that in more recent studies, children-especially girls—spent less time playing with sex-typed toys. We speculated in our paper that this effect might reflect social pressures in recent times for children to be less gender-typical in their behavior.

We would recommend that Davis and Hines replicate our analysis, though because not all studies yield information on the mean $( \pm \mathrm{SD})$ duration of play, this will limit the number of studies used in their analysis. It would be interesting to see whether they find a correlation between the year a study was published and the time spent by children in sex-typed toy play. Such an analysis could be especially interesting if it finds that the effect varies across the four different assessment methods examined by Davis and Hines. It could be that different methods (free play, visual preference, etc.) yield different results, analogous to the way that sex difference in visuospatial ability is reliably seen using the three-dimensional mental rotation task, rather than visuospatial tasks in general.

\section{References}

Davis, J. T., \& Hines, M. (2020). How large are gender differences in toy preferences? A systematic review and meta-analysis of toy preference research. Archives of Sexual Behavior, 49(2), 373-394.

Todd, B. K., Fischer, R. A., Di Costa, S., Roestorf, A., Harbour, K., Hardiman, P., \& Barry, J. A. (2018). Sex differences in children's toy preferences: A systematic review, meta-regression, and meta-analysis. Infant and Child Development, 27(2), e2064.

Publisher's Note Springer Nature remains neutral with regard to jurisdictional claims in published maps and institutional affiliations.
John A. Barry

john.barry@ucl.ac.uk

1 Department of Clinical, Educational and Health Psychology,

Faculty of Brain Sciences, University College London, 1-19

Torrington Place, London WC1E 6BT, UK

2 Department of Psychology, School of Social Sciences, City

University of London, Northampton Square, London, UK 